\title{
Perturbative Gluon Shadowing in Heavy Nuclei
}

\author{
K.J. Eskola, ${ }^{1,2}$ Jianwei Qiu ${ }^{3}$ and Xin-Nian Wang ${ }^{1}$ \\ ${ }^{1}$ Nuclear Science Division, Mailstop 70A-3307, Lawrence Berkeley Laboratory, \\ University of California, Berkeley, CA 94720, USA \\ ${ }^{2}$ Laboratory of High Energy Physics, P.O. Box 9, (Siltavuorenpenger 20C) \\ SF-00014 University of Helsinki, Finland. \\ ${ }^{3}$ Department of Physics and Astronomy, Iowa State University, \\ Ames, IA 50011, USA
}

July 23, 1993

\begin{abstract}
We study how much gluon shadowing can be perturbatively generated through the modified QCD evolution in heavy nuclei. The evolution of small- $x$ gluons is investigated within the semiclassical approximation. The method of characteristics is used to evaluate the shadowed distributions in low- $Q$ and small- $x$ region. In solving the modified evolution equation, we model in simultaneously fusions from independent constituents and from the same constituent, both in a proton and in a large loosely bound nucleus of $A \sim 200$. In addition to the actual distributions at small $x$, we study the ratios of the distributions at an initial scale $Q_{0}=2 \mathrm{GeV}$, and show that a strong nuclear shadowing can follow from the modified QCD evolution.
\end{abstract}

${ }^{*}$ This work was supported by the Director, Office of Energy Research, Division of Nuclear Physics of the Office of High Energy and Nuclear Physics of the U.S. Department of Energy under Contract No. DE-AC03-76SF00098. 
The semihard gluonic subprocesses are expected to play an essential role in the formation of high energy densities in heavy ion collisions at collider energies [1, 2, 3, 付. However, there are many theoretical uncertainties in modeling these QCD processes. One of the major ones, nuclear gluon shadowing, comes from the unknown initial gluon distributions at small $x$. Unlike for the quark and antiquark distributions, there are no direct experimental data for the gluons in nuclei. Getting theoretical control over the nuclear gluon shadowing is therefore a very urgent and important issue. The purpose of this Letter is to study how much gluonic shadowing is generated perturbatively through the modified QCD evolution [5, 6] in heavy nuclei.

"Shadowing" in the context of the deep inelastic $l A$-scattering refers to the measured depletion of the nuclear structure function $F_{2}^{A}$ at small $x_{\mathrm{Bj}}$, as compared to $F_{2}$ of unbound nucleons [7]. The same kind of depletion at small $x$ is expected to happen also in the nuclear gluon distributions. During the recent years there have been many efforts to explain the measured nuclear shadowing of quarks and antiquarks [8- 15] but for gluons the situation is still inconclusive. Once the nuclear parton distributions are known at an initial scale $Q_{0}$, the QCD-evolution to larger $Q$ can be computed [0, [16, 17]. The problem is how to get input, theoretically or experimentally, for the nuclear gluon distributions at $Q_{0}$, and to understand the reliability of QCD-evolution for the proper range of $x$ - and $Q$-values.

Shadowing-phenomenon is also predicted to happen in protons. In this case, "shadowing" refers to the depletion of the actual parton distributions, and is caused by the fusions of overcrowding gluons at very small $x$. This mechanism proceeds through perturbative QCD-evolution as formulated in [5, 6]. It has been shown by Collins and Kwieciński that the singular gluon distributions actually saturate due to the fusions [18].

In this Letter our basic idea is quite straightforward. We first compute the gluon shadowing in a proton, by using techniques introduced in [18] for solving the small$x$ evolution including gluon recombination. Then we apply the same mechanism of recombining gluons to heavy nuclei and study to what extent nuclear shadowing is generated perturbatively through the QCD-evolution at $Q_{0}=2 \mathrm{GeV}$.

At small values of $x$, leading order QCD evolution equation predicts that the number of gluons becomes extremely large. It has been known [5, 6] that for sufficiently small values of $x$ and/or of $Q^{2}$, the total transverse area occupied by the gluons will be larger than the transverse area of a hadron, so that the interaction between gluons can no longer be neglected. Such gluon recombination results in a modification of the QCD evolution equations. In the limit of small- $x$ the modified QCD evolution 
equation can be cast in the form [5, 6]

$$
\partial_{y} \partial_{t} G(y, t)=c G(y, t)-\lambda \exp \left(-t-\mathrm{e}^{t}\right)[G(y, t)]^{2},
$$

where $y=\ln (1 / x), t=\ln \left[\ln \left(Q^{2} / \Lambda_{\mathrm{QCD}}^{2}\right)\right], G(y, t)=x g\left(x, Q^{2}\right)$ and $c=12 /\left(11-2 N_{f} / 3\right)$ with $N_{f}$ the number of quark flavors.

Strength of the gluon recombination is controlled by the factor $\lambda$, originating from two possible sources. The two fusing gluon ladders, which couple 4 gluons to 2 gluons, can arise either from independent constituents of proton/nucleus or from the same one, as discussed in [6, 18, 21]. We will refer to the former case as "independent" and to the latter as "non-independent" fusion. Since recombinations from both sources happen simultaneously, we divide the parameter $\lambda$ into two parts:

$$
\lambda=\lambda_{\mathrm{I}}+\lambda_{\mathrm{II}}
$$

where $\lambda_{\mathrm{I}}$ corresponds to the independent recombination and $\lambda_{\mathrm{II}}$ to the non-independent one.

Let us first study the two sources of recombination within the models for twogluon densities given in ref. [6]. In a proton, the strength of the independent fusion then takes the form

$$
\lambda_{\mathrm{I}}=\frac{2}{3} \frac{1}{\pi R_{p}^{2}} \cdot \frac{\pi^{3} c^{2}}{2 \Lambda_{\mathrm{QCD}}^{2}},
$$

where $R_{p} \sim 1 \mathrm{fm}$ is the radius of a proton.

The magnitude of the non-independent fusion of the gluon ladders can be estimated as

$$
\lambda_{\mathrm{II}} \approx \frac{16}{81} \frac{1}{\pi\left(2 / Q_{i}\right)^{2}} \cdot \frac{\pi^{3} c^{2}}{2 \Lambda_{\mathrm{QCD}}^{2}},
$$

where we have made a simplification by fixing the initial $x$ of the valence quark to $x_{i} \sim 1$. We also approximate the scale of the initial valence quark by $Q_{i} \sim 2 \mathrm{GeV}$.

Let us then consider a large loosely bound nucleus. Naturally, both types of fusions are still there but only for the independent one an $A^{1 / 3}$-scaling arises. In this case

$$
\lambda_{\mathrm{I}}^{A}=\frac{9}{8} \frac{A}{\pi R_{A}^{2}} \cdot \frac{\pi^{3} c^{2}}{2 \Lambda_{\mathrm{QCD}}^{2}},
$$

where the nucleus is taken to be a sphere with a sharp surface at $R_{A}=1.12 A^{1 / 3} \mathrm{fm}$. The strength of the non-independent fusion remains the same as in the case of a free proton: $\lambda_{\mathrm{II}}^{A}=\lambda_{\mathrm{II}}$.

It is interesting to notice how the relative contributions of the two types of recombination will change when going from a proton to a nucleus of $A \sim 200: \lambda_{\mathrm{II}} / \lambda_{\mathrm{I}} \approx 7.6$ 
and $\lambda_{\mathrm{II}}^{A} / \lambda_{\mathrm{I}}^{A} \approx 1.0$. Thus the non-independent fusion is clearly dominant in a free proton while in a large nucleus the contributions from both types are of the same order. As a result, parton recombination is strongly enhanced in a heavy nucleus.

In order to solve Eq. 11 exactly by integration, one would need the initial distribution either at fixed $y_{0}$ or $t_{0}$ and the derivatives along a boundary line $\left(y, t_{0}\right)$ or $\left(y_{0}, t\right)$, respectively. However, since the expression for the non-linear term in Eq. 1 is not valid for the regions where $x$ is large, or where both $x$ and $Q$ are very small, the natural boundary condition at $x=1$ ( or $y_{0}=0$ ) is not suitable here. In addition, since we do not have sufficient information on other boundary lines, we cannot solve Eq. 11 by direct integration. Instead, with the semiclassical approximation [5], we are going to adopt the idea introduced in [18 to use the method of characteristics, so that we can avoid the region III (see discussion later).

The semiclassical approximation corresponds to neglecting the second order derivative term, $\partial_{y} \partial_{t} \ln (G)$, which leaves us with the evolution equation as

$$
\partial_{y} z(y, t) \partial_{t} z(y, t)=c-\lambda \exp \left[-t-\mathrm{e}^{t}+z(y, t)\right]
$$

where $z(y, t)=\ln [G(y, t)]$. The above equation can then be cast and solved in the form of a set of characteristic equations as shown in detail in [18].

The evolution of gluon distribution in a proton and in a nucleus is similar, so let us first consider the general idea, as illustrated in Fig. 1. We divide the $(y, t)$-plane into three regions. In region $I$ for $y \leq y_{0}\left(x \geq x_{0}\right)$, we expect the traditional, non-corrected Altarelli-Parisi (AP) evolution [19] to hold down to scales $Q \sim 1 \mathrm{GeV}$. In region $I I$ with $y \geq y_{0}\left(x \leq x_{0}\right)$, the evolution of the gluon distribution is then approximately given by Eq. 6. The initial values of the gluon distribution and its $t$ - and $y$-derivatives are determined numerically at $\left(y_{0}, t_{\min } \leq t \leq t_{0}\right)$ from the singular gluon distribution of the CTEQ-collaboration [20], which is obtained with a lower initial $Q$-value to provide the necessary $t$-dependence of the boundary condition at $y_{0}$, and a larger $x$-cut to ensure the validity of AP evolution in region $I$. The evolution in the region $I I$ is finally terminated at $t=t_{0}$, corresponding to $Q_{0}=2 \mathrm{GeV}$. Notice that the characteristics approach the $t_{0}$-line from below; the lowest scale we have to go down to is about $Q=1.25 \mathrm{GeV}$, corresponding to $t_{\text {min }}$. At these scales QCD perturbation theory should be still valid in region $I$. In region $I I I$ with extremely large $y$ (small $x$ ) and/or small $t$ we do not expect our analysis to be valid anymore, since the higher order terms in the evolution equation will become important.

Before performing the actual evolutions, we have to consider how to choose the boundary $y_{0}\left(=-\ln \left(x_{0}\right)\right)$ for a proton and a nucleus, and how to conserve momentum. 
For a proton, we assume that the recombinations start to be effective at $x \sim x_{0} \sim$ 0.01 , which is consistent with [18, 21]. We can use the results from global fitting, like CTEQ, to constrain $x_{0}$. In fact, we will see that with $x_{0}=0.01$ the shadowed gluons deviate considerably from the CTEQ gluons only after $x<0.001$, so the choice for $x_{0}$ seems to be reasonable, and we do not expect the results to be very sensitive to small changes of $x_{0}$.

As explained above, the gluon recombination is strongly enhanced in heavy nuclei and it starts at somewhat larger values of $x$ than in protons. The corresponding boundary line $x_{0}^{A}$ for a nucleus is approximately determined by the relative magnitude of the evolution terms in Eq. ㄱ: $G_{A}\left(x_{0}^{A}\right) \sim G\left(x_{0}\right) \lambda_{A} / \lambda$, so that the relative contribution from the gluon fusion in a nucleus is about the same as in a nucleon. This gives $x_{0}^{A} \sim 0.05-0.1$. This range of $x_{0}^{A}$ is also supported by other studies [17].

Let the original fraction of momentum in gluons be $f_{0}=\int_{0}^{1} d x x g_{\mathrm{CTEQ}}\left(x, Q_{0}^{2}\right)$. In the case of a proton, shadowing in the region $I I$ changes the gluonic momentum typically by less than a per cent, which we can clearly neglect as a small overall change.

Perturbative shadowing reduces the gluonic momentum more in a nucleus than in a proton. Assumed that the momentum fraction of gluons is conserved, there must be a corresponding enhancement in the region $I I$. In addition to this, we also take into account a possible momentum transfer from quarks and antiquarks to the gluons. Here we consider nuclei with $A \sim 200$, for which we expect an overall increase in the fraction of the momentum, $\epsilon_{A}$, to be only about $4 \%$ [9, 10, 17]. We combine these two sources of the momentum flow, which results in solving $a_{A}$ iteratively from

$$
\left.\int_{0}^{x_{0}^{A}} d x x g\left(x, Q_{0}^{2}\right)\right|_{C}+a_{A} \int_{x_{0}^{A}}^{1} d x x g_{\mathrm{CTEQ}}\left(x, Q_{0}^{2}\right)=f_{0}\left(1+\epsilon_{A}\right),
$$

with the condition $C: g_{A}\left(x_{0}^{A}, Q_{0}^{2}\right)=a_{A} g_{\mathrm{CTEQ}}\left(x_{0}^{A}, Q_{0}^{2}\right)$ on the boundary. Typically, $a_{A} \sim 10 \%$ for $A \sim 200$.

Let us now turn to the results, presented in Figs. 2. In Fig. 2a, nucleon and effective nuclear gluon distributions for a nucleus of $A=200$ are compared with the input CTEQ gluon distribution at $Q_{0}=2 \mathrm{GeV}$. Notice the $\sim 20 \%$ uncertainty in the nuclear case resulting from varying $x_{0}^{A}$ from 0.05 to 0.1 . To demonstrate the formation of strong perturbative nuclear shadowing, corresponding to the relative depletion of gluon distributions in a nucleus, we plot the ratio $G_{A}\left(x, Q_{0}^{2}\right) / G\left(x, Q_{0}^{2}\right)$ in Fig. $2 \mathrm{~b}$. Notice also that as $x$ decreases, gluon distribution in a proton increases much faster, or shows the sign of saturation at a much smaller $x$ than that in a nucleus. Therefore, as shown in Fig. $2 \mathrm{~b}$ the ratio saturates only when the gluons in a proton do so. Thus, 
saturation of the perturbative nuclear shadowing reflects actually the behavior of the gluons in a proton. As a main result, we conclude that, due to the enhanced gluon recombination in a heavy nucleus, a $\sim 50 \%$ nuclear shadowing in small- $x$ region is generated perturbatively through the modified QCD evolution, accompanied by a $\sim 10 \%$ antishadowing from the momentum conservation.

As seen more clearly in Fig. 2b, with $x_{0}^{A}=0.1$ there is a slight deviation in the initial derivatives of the gluon distributions determined from the region $I$ as compared to what can be determined from Eq. 6 from the region $I I$. This in turn is a reflection of an apparent fact that eventually one cannot apply the small- $x$ approximation at too large $x$. Letting $x_{0}^{A} \sim 0.1$, we are really pushing the small- $x$ evolution equation to its limit; surely beyond this point the Eqs. 1 and 6 cannot be applied without additional correction terms. However, taking the initial conditions from the "known" region $I$, as we do, should improve the analysis and reduce the uncertainty in the small- $x$ region. From Fig. $2 \mathrm{~b}$ it is seen that we cannot make conclusive claims about the "beginning" of nuclear shadowing. However, since the result with $x_{0}^{A}=0.1$ does not differ considerably from the result with $x_{0}^{A}=0.05$, we believe our result shows the correct order of magnitude of the perturbative shadowing at very small $x$.

It is clear that the absolute strengths of the $\lambda$ 's depend on the models assumed for the two-gluon densities in a proton and in a nucleus. However, we do not expect our qualitative results for the nuclear shadowing to change very much with different details. One may also question what happens to nuclear shadowing, if the initial gluons diverge more strongly(weakly) when $x \rightarrow 0$ than CTEQ gluon distribution used here. In that case, the recombinations would be enhanced(suppressed) both in a proton and in a heavy nucleus. However, the result for nuclear shadowing, $G_{A}\left(x, Q_{0}^{2}\right) / G\left(x, Q_{0}^{2}\right)$, is not extremely sensitive to the small- $x$ behavior of the input gluon distribution because we use only the part with $x>0.01$, which has been relatively well-tested experimentally. This question will be studied in more detail elsewhere [23].

We would like to comment briefly on the general consequences of our result for perturbative nuclear shadowing. The semihard processes with typical scales $Q \sim$ a few $\mathrm{GeV}$ involve $x \sim Q / \sqrt{s}$. In heavy ion collisions at $\sqrt{s}=200 \mathrm{GeV}$, the $x$ 's in the semihard processes will be larger than 0.01, so these processes rather probe the onset of perturbative nuclear shadowing than the region of saturation. On the contrary, in collisions with $\sqrt{s}$ in the TeV range, the semihard processes will happen at $x$ 's typically smaller than $10^{-3}$, and are therefore affected considerably more by the perturbative shadowing. Examples of the possible effects on minijet production 
can be found in [2, 17, 22]. Other processes clearly suppressed by the nuclear gluon shadowing at very high energies are heavy quark and their bound state production. Also the production of total transverse energy and energy density will be suppressed, as compared to the predictions with non-shadowed gluons [2, 22]. Through this, the thermalization of the possibly formed quark-gluon plasma is also slowed down, in which case the thermal electromagnetic signals are suppressed. In order to make precise predictions for these processes, nuclear shadowing has to be studied at scales $Q>2 \mathrm{GeV}$. The scale dependence of nuclear gluon shadowing is an interesting question to which we will return in the future [23].

To conclude, we have considered the perturbative aspects of the nuclear modifications to the gluon distributions. As we have shown here, a strong nuclear shadowing is generated through the modified QCD evolution, and it may well be the dominant mechanism for the small- $x$ modifications. We emphasize that the use of the method of characteristics is necessary to avoid the region $I I I$ where even the modified evolution equation is not expected to be valid. We feel we now have more quantitative control over the nuclear gluon distribution at small $x$, based on perturbative QCD. We believe this study could serve as an interesting starting point for more detailed calculations of nuclear gluon shadowing and its consequences in ultra-relativistic heavy ion collisions.

Acknowledgements. We acknowledge helpful discussions with J. Owens, R. Vogt and S. Gavin. KJE is grateful to Emil Aaltonen foundation, Magnus Ehrnrooth foundation and Suomen Kulttuurirahasto for partial financial support. JWQ thanks the Nuclear Science Division at Lawrence Berkeley Laboratory for hospitality while part of this work was completed. This work was supported by the U.S. Department of Energy under contract No. DE-AC03-76SF00098, and in part by the Grant Nos. DE-FG02-87ER40371 and DE-FG02-92ER40730, and by the Texas National Research Laboratory Commission.

\section{References}

[1] K. Kajantie, P. V. Landshoff and J. Lindfors, Phys. Rev. Lett. 59 (1987) 2527;

K. J. Eskola, K. Kajantie and J. Lindfors, Nucl. Phys. B323 (1989) 37.

[2] X.-N. Wang and M. Gyulassy, Phys. Rev. D44 (1991) 3501; ibid. D45 (1992) 844; Phys. Rev. Lett. 68 (1992) 1480. 
[3] K. Geiger and B. Müller, Nucl. Phys. B369 (1992) 600; K. Geiger, Phys. Rev. D47 (1993) 133.

[4] I. Kawrakow, H.-J. Möhring and J. Ranft, Nucl. Phys. A544 (1992) 471c.

[5] L. V. Gribov, E. M. Levin and M. G. Ryskin, Phys. Rep. 100 (1983) 1; Nucl. Phys. B188 (1981) 555; Zh. Eksp. Teor. Fiz. 80 (1981) 2132.

[6] A. H. Mueller and Jianwei Qiu, Nucl. Phys. B268 (1986) 427.

[7] EM Collaboration, M. Arneodo et al., Nucl. Phys. B333 (1990) 1; NM Collaboration, P. Amaudruz et al, Z. Phys. C51 (1991) 387; E665 Collaboration, M. R. Adams et al., Phys. Rev. Lett. 68 (1992) 3266; Phys. Lett. B287 (1992) 375.

[8] N.N. Nikolaev and V.I. Zakharov, Phys. Lett. B55 (1975) 297; Z. Phys. C49 (1991) 607; Phys. Lett. B260 (1991) 414; Z. Phys. C53 (1992) 331.

[9] L. L. Frankfurt and M.I. Strikman, Phys. Rep. 160 (1988) 237; Nucl. Phys. B316 (1989) 340; L. L. Frankfurt, M. I. Strikman and S. Liuti, Phys. Rev. Lett. 65 (1990) 1725.

[10] F. E. Close, J. Qiu and R.G. Roberts, Phys. Rev. D40 (1989) 2820.

[11] P. Castorina and A. Donnachie, Z. Phys. C45 (1989) 141.

[12] S. J. Brodsky and H. J. Lu, Phys. Rev. Lett. 64 (1990) 1342.

[13] J. Kwieciński, Z. Phys. C45 (1990) 45.

[14] V. Barone et al, IKP (Theorie) Forschungszentrum Preprint KFA-IKP(TH)1992-13.

[15] S. Kumano, Phys. Lett. B298 (1993) 171.

[16] Jianwei Qiu, Nucl. Phys. B291 (1987) 746.

[17] K. J. Eskola, LBL-Preprint 32339, Nucl. Phys. B, in press.

[18] J. Collins and J. Kwieciński, Nucl. Phys. B335 (1990) 89.

[19] G. Altarelli and G. Parisi, Nucl. Phys. B126 (1977) 298.

[20] J. Botts, et. al., Phys. Lett. B304 (1993) 159. 
[21] J. Kwieciński, A. D. Martin, W. J. Stirling and R. G. Roberts, Phys. Rev. D42 (1990) 3645.

[22] K.J. Eskola, Z. Phys. C51 (1991) 633.

[23] K.J. Eskola, Jianwei Qiu and X.-N. Wang, in preparation.

\section{Figure Captions}

Fig. 1. The evolution plane. In the region $I$ the traditional AP-equations are expected to be valid. Both $x$ - and $Q$-dependence in this region form the initial conditions for the evolution in the small-x (large $y$ ) region $I I$. Examples of the characteristics of the Eq. 6 in the region $I I$ are shown.

Fig. 2. a. The gluon distributions $x g\left(x, Q_{0}^{2}\right)$ at $Q_{0}=2 \mathrm{GeV}$ vs. $x$. The result for proton is labeled by $x_{0}$, and the results for $A \sim 200$ by $x_{0}^{A}$, respectively. The CTEQ

gluon distribution [20] is labeled by "CTEQ". b. The ratio $x g_{A}\left(x, Q_{0}^{2}\right) / x g\left(x, Q_{0}^{2}\right)$ of the shadowed gluon distributions vs. $x$, demonstrating a strong perturbative nuclear shadowing in heavy nuclei. 


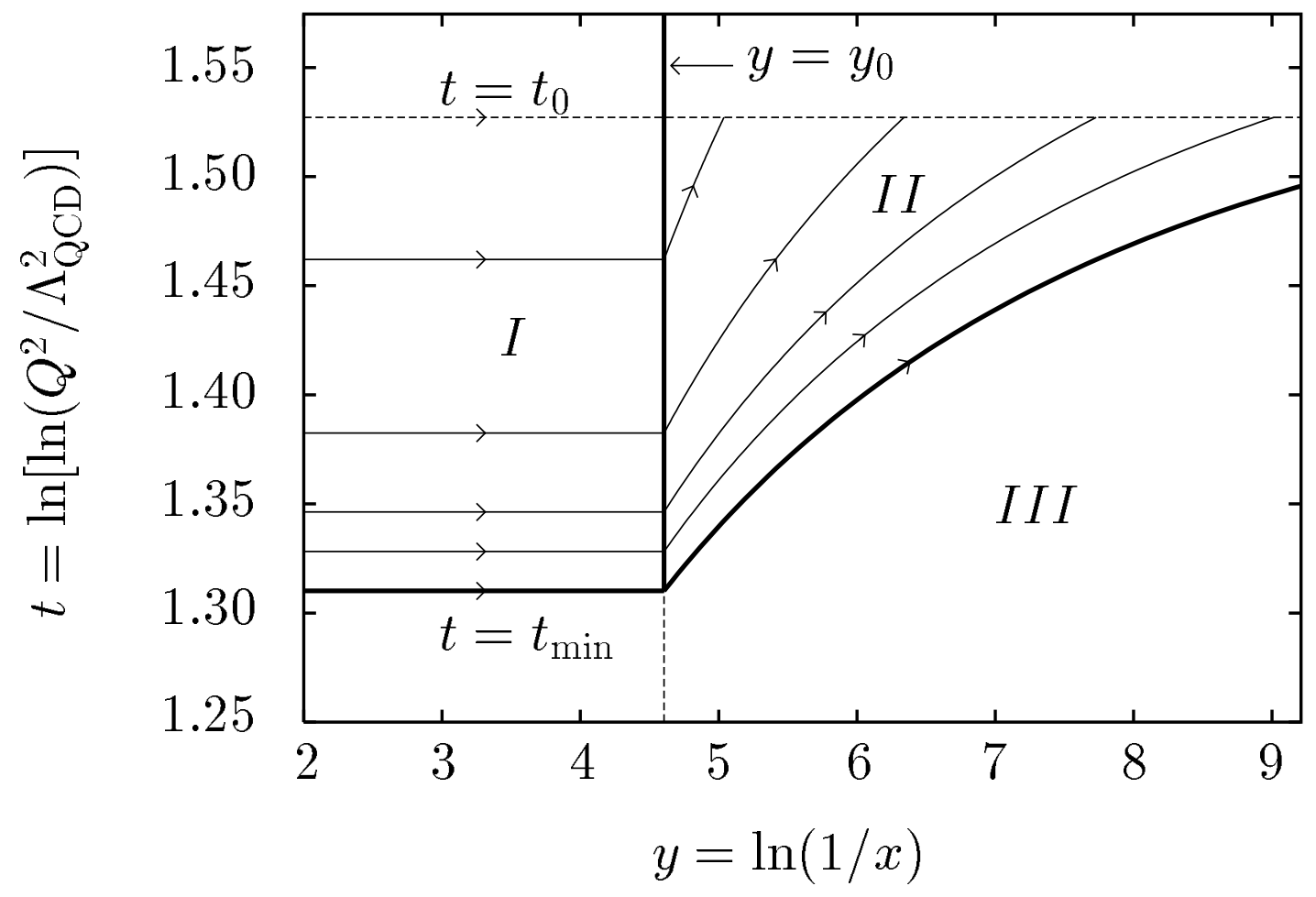

Fig. 1 
Figs. 2 a,b

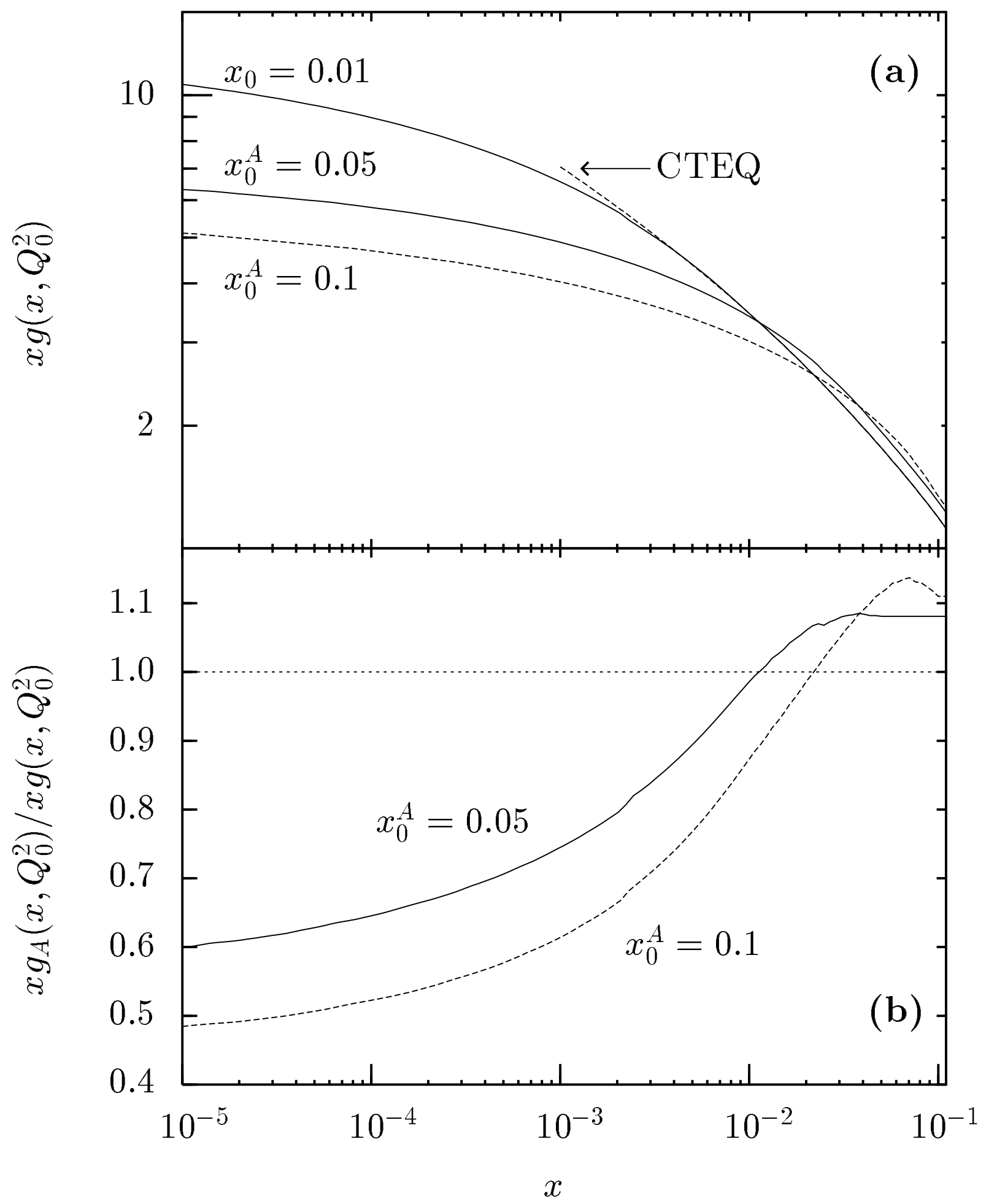

